\title{
Neonatal cerebral blood flow velocity responses to changes in posture
}

\author{
M Y Anthony, D H Evans, M I Levene
}

\begin{abstract}
Maintaining a constant cerebral blood flow during a change in cerebral perfusion pressure is known as autoregulation. The integrity of this phenomenon is considered to be important in preventing cerebral lesions in preterm infants. A study was carried out using Doppler ultrasound measurements of cerebral blood flow velocities (CBFV) as an indicator of alterations in cerebral haemodynamics. CBFV were recorded on a beat to beat basis over 60 second epochs, during which time the cerebral perfusion pressure was changed by rapidly altering the infants' posture from horizontal to either $20^{\circ}$ head up or head down. An informative response in CBFV was considered to be either (a) a uniphasic, immediate, passive alteration in velocity occurring with the change in posture and without a subsequent change or (b) a biphasic response of an initial change in CBFV followed within 20 seconds by a second response. This latter response is considered to be consistent with autoregulatory activity. A total of 501 epochs in 60 neonates of gestational age 24-41 weeks was analysed. It was shown that any one infant can make either response, but the reliability of making an active, biphasic response increases with increasing gestational age.

(Arch Dis Child 1993; 69: 304-308)
\end{abstract}

A failure in the control of cerebral haemodynamics, or failure of autoregulation, is thought to be an important aetiological factor in the development of various cerebral lesions in preterm infants. ${ }^{12}$ Several studies, using various methods of measurement, have tried to show a passive cerebral circulation in response to presumed alterations in cerebral perfusion pressure. ${ }^{3-9}$ Many of these studies have been hampered by a lack of direct and continuous measurements of cerebral haemodynamics during the imposition of the stimuli. Autoregulatory responses in animal models and humans occur swiftly, within seconds, and therefore many studies are unable to detect true autoregulatory responses. ${ }^{10-14}$ We have previously described an on line Doppler system which uses a small pulse wave transducer which can be secured to the skin over the temporal bone of a neonate to insonate the middle cerebral artery from a fixed position and constant angle for 60 second epochs. ${ }^{15-17}$ During this period of constant beat to beat observation the posture of the infant can be altered and the changes in the cerebral blood flow velocities (CBFV) caused by the change in cerebral perfusion pressure observed until the end of the epoch. The resulting tracings are analysed for either a simple, uniphasic, presumably passive alteration occurring with the stimulus, or for evidence of a second opposing, biphasic, presumably active alteration in CBFV occurring after the first passive change. This study does not make any assumptions about the relation of Doppler velocity readings to cerebral blood flow.

\section{Subjects and methods}

\section{DOPPLER ULTRASOUND}

The Doppler ultrasound system has been described previously. ${ }^{15-17}$ A 4 or $6 \mathrm{MHz}$ Doptek pulsed wave, button shaped transducer was secured onto the skin over the infant's temporal bone to insonate the ipsilateral middle cerebral artery. A soft collar of Stomahesive (Squibb, Hounslow, Middlesex) was applied to the skin around the probe and the probe secured to the collar with collodion. The Stomahesive protects the infant's skin from the collodion. The sample volume of the Doppler signal was adjusted to optimise the signal and once in place the transducer remained fixed for several hours before being electively removed.

The probe was connected via a fine, lightweight, coaxial cable to the Doppler unit, which in turn was connected to a Nimbus computer and digital signal processing system. ${ }^{17}$ The computer automatically activated the Doppler signal to record the velocity of the blood cells within the middle cerebral artery for a 60 second period every 20 minutes. The data were analysed, transferred to disk, and the graphs of the systolic, mean, and diastolic velocities displayed after each epoch on the Nimbus screen; these were later printed onto paper for subsequent analysis. The unit is mobile and was brought to the cot side of the infant.

\section{PATIENTS}

Two groups of infants were studied, one requiring neonatal intensive care and the other a group of normal, healthy, term infants nursed on the postnatal wards.

All the infants receiving intensive care had indwelling arterial lines. Their blood pressure was monitored continuously from the arterial cannula and during the 60 second epoch of velocity data acquisition the simultaneous beat to beat blood pressure was also recorded by the Nimbus system from the blood pressure monitor. All had regular arterial blood gas 
measurements and all had regular cerebral ultrasound examinations. These infants were routinely nursed under an overhead heater on a mattress with a tilting mechanism. The Doppler probe was attached as described and an epoch of data gathered to ensure a satisfactory recording. During subsequent epochs, at timed intervals from the start of the recording, the mattress was tilted, either from the horizontal to $20^{\circ}$ head up or $20^{\circ}$ head down. The direction and timing of the movement were noted so that the point of changing the cerebral perfusion pressure could be marked onto the printed graph of the velocity data. Each infant was studied for as long as a good signal was maintained and for as long as the study did not interfere with the infant's clinical management. Some infants were studied on more than one occasion on consecutive days.

The healthy term infants were placed in a baby chair with a tilting mechanism. The probe was attached as described and an epoch run to check the tracing. On subsequent recordings the chair back was tilted either from the relatively upright to lying back, or from the lying back position to a more upright position a movement of approximately $20^{\circ}$. The healthy infants were studied for a maximum period of one hour 20 minutes, in which a total of four recordings could be made.

The study was approved by the hospital's ethics committee and informed consent was obtained from at least one parent.
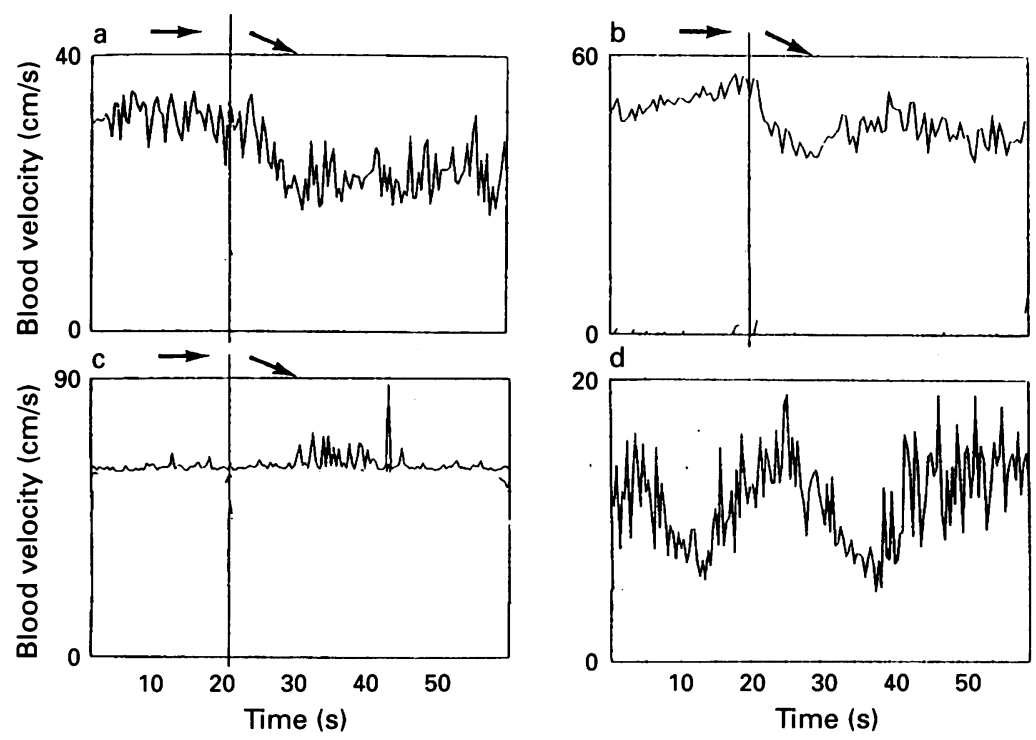

Figure 1 Graphs of systolic velocity against time showing: (a) a uniphasic response to a change in posture; (b) a biphasic response to a change in posture; (c) no change; and (d) a cyclical pattern of CBFV with superimposed marked beat to beat variability.

Effect of change in posture in the studied infants by gestational age. The median (range) percentage response is shown

\begin{tabular}{|c|c|c|c|c|}
\hline & \multicolumn{4}{|c|}{ Gestational age (weeks) } \\
\hline & \multicolumn{3}{|c|}{ Intensive care } & \multirow{2}{*}{$\begin{array}{l}\text { Healthy full term } \\
\begin{array}{l}\text { Median } 39 \\
(n=10)\end{array}\end{array}$} \\
\hline & $\begin{array}{l}\leqslant 26 \\
(n=13)\end{array}$ & $\begin{array}{l}>27<32 \\
(n=29)\end{array}$ & $\begin{array}{l}\geqslant 32 \\
(n=8)\end{array}$ & \\
\hline $\begin{array}{l}\text { Equivocal/cycling } \\
\text { No response } \\
\text { Uniphasic } \\
\text { Biphasic }\end{array}$ & $\begin{array}{r}35(0-71) \\
33(0-63) \\
3(0-50) \\
11(0-50)\end{array}$ & $\begin{array}{r}20(0-86) \\
22(0-71) \\
0(0-60) \\
12(0-87)\end{array}$ & $\begin{array}{c}40(0-54) \\
23 \cdot 5(0-60) \\
0(0-20) \\
20(0-50)\end{array}$ & $\begin{array}{c}25(0-33) \\
0(0-33) \\
0(0-50) \\
66(0-100)\end{array}$ \\
\hline
\end{tabular}

Paper printouts of the beat by beat systolic velocity data were later studied for one of four responses to the change in posture (see fig 1): (a) a sudden change in CBFV, within five seconds of the change in posture, in either direction, but no second change within 20 seconds of the first (a uniphasic response); (b) a sudden change in $\mathrm{CBFV}$, within five seconds of the change in posture, in either direction followed by a second change in velocity within 20 seconds of the first (a biphasic response); (c) no change in CBFV; and (d) an equivocal tracing with regular cycling CBFV across the epoch or an uninterpretable trace, such as large beat to beat variability.

\section{Results}

A total of 60 infants was studied. Fifty infants required intensive care. The median (range) gestational age was $28(24-37)$ weeks and the birth weight was $1150(580-2820) \mathrm{g}$. The number of epochs for each infant was 8 (3-31). Ten healthy term infants were studied; their gestational age was 39 (37-41) weeks, and birth weight 3070 (2820-3910) g. Each infant was studied for a maximum of four epochs. All infants were less than 1 week old at the time they were studied. A total of 501 epochs was recorded.

Previously reported cyclical and beat to beat velocity variations made the interpretation of the velocity changes difficult in some infants. ${ }^{16}$ Before the analysis of the traces clear definitions of the four types of patterns were made so that it was possible for two independent observers to agree on the interpretations. Any traces where doubt existed were discarded as equivocal. There was no difference in the number of uninterpretable traces with gestational age.

Of the four possible responses of the CBFV to a change in posture the percentage of epochs showing a particular response in an infant was derived from the total number of epochs obtained for that infant. The table gives the percentage responses for the infants undergoing intensive care, divided into gestational age groupings, and the healthy full term infants.

The plot of percentage biphasic responses against gestational age (fig 2) shows an increasing percentage of biphasic response with increasing gestational age $(p<0 \cdot 0001)$.

Of the preterm infants studied, neither a high arterial carbon dioxide tension $\left(\mathrm{PaCO}_{2}\right)>6.0$, nor a mean arterial blood pressure $<25 \mathrm{~mm} \mathrm{Hg}$ influenced the percentage biophasic response. Interestingly, biphasic and uniphasic responses could occur in consecutive epochs with all other parameters remaining constant. The infants who sustained cerebral lesions were distributed evenly across the high percentage biphasic responders and low percentage biphasic responders.

\section{Discussion}

The concept of autoregulation of the cerebral blood supply was put forward in the 1930s by 


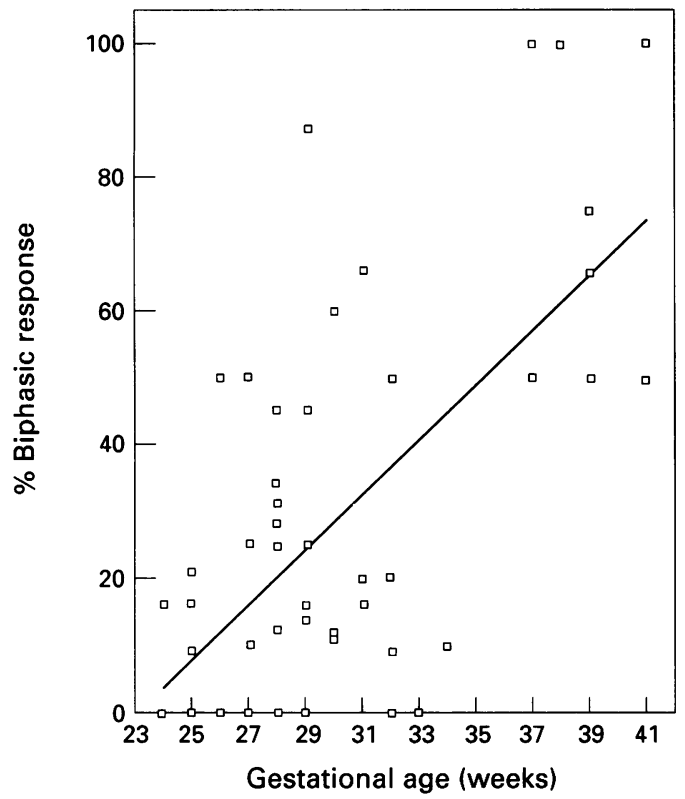

Figure 2 Graph of \% biphasic response against gestational age; $y=-94 \cdot 8+4 \cdot 11 x, p<0 \cdot 0001$.

Fog. ${ }^{18}{ }^{19} \mathrm{He}$ observed changes in the pial vessels of cats in response to changes in systemic blood pressure. The idea was refined in 1959 by Lassen to indicate a consistent cerebral blood flow with a changing cerebral perfusion pressure (cerebral perfusion pressure $=$ mean arterial pressure minus intracranial pressure). ${ }^{20}$

Various studies in adult humans have shown the cerebral blood flow to be maintained within a range of mean arterial pressure of 50-175 $\mathrm{mm} \mathrm{Hg}$. Beyond these limits the autoregulatory controls fail ${ }^{101321}$ and it is known that many variables will influence autoregulatory changes. ${ }^{14}$ 21-23

The site and speed of autoregulatory responses have been investigated in human adults, cats, dogs, rabbits, and baboons. ${ }^{10} 14$ 24-34 Species variability occurs, but it is evident that unlike other organ systems the large cerebral arteries, including the middle cerebral artery, and the small arteries and arterioles contribute to the changing vascular resistance in response to various stimuli; therefore, in Doppler studies, it is important not to assume that the cross sectional area of cerebral arteries, such as the middle cerebral artery, remains constant. These studies have also shown that a change in cerebral perfusion pressure will cause an immediate passive alteration in the blood flow to the head, which is followed within three to seven seconds by a second corrective change. In some species a steady state is resumed within one minute, although in baboons it has been shown that the corrective changes initiated within three to seven seconds continue to oscillate, re-establishing an equilibrium within about four minutes. ${ }^{33}$

Human neonates have been the subject of much speculation about their ability to autoregulate cerebral blood flow. Methods used as indicators of cerebral blood flow have included labelling with xenon-133, jugular occlusive plethysmography, and Doppler ultrasound. ${ }^{3-9}$ The published data must be viewed with caution as measurements of cerebral blood flow or CBFV and blood pressure have often been made intermittently or between subjects. These have not been continuous observations, within one subject, over the three to seven seconds after a change in cerebral perfusion pressure, the time scale in which autoregulatory changes are expected to occur. Other factors will influence readings of $\mathrm{CBFV}$ taken at different times and between subjects, notably $\mathrm{PaCO}_{2}$ and the packed cell volume. In some studies measurements were made in sick, acidotic infants, some of whom were in extremis. The value of such observations to the 'normal' preterm infant is questionable. Some studies use cuff inflation methods of measuring the systemic blood pressure. The reliability of this method is not good and the method can only be used intermittently.

In our study the stimulus used to elicit an autoregulatory response was a change in posture to provoke a change in cerebral perfusion pressure. All the infants undergoing intensive care had indwelling arterial catheters for clinical purposes. Although the infants receiving intensive care were obviously sick, they were in a stable clinical condition and none could be described as being in extremis. Spontaneous, sustained changes in blood pressure were rare and could not be reliably used to study autoregulatory responses. Tipping the mattresses altered the blood pressure reading in some infants but the true alteration in pressure at the cerebral level could not be determined by a peripheral arterial line and movable transducer. It is known that in adults a change in posture of the order imposed on the infants causes a $20 \%$ change in cerebral perfusion pressure. ${ }^{21}$ In adults the alteration in cerebral perfusion pressure is ameliorated by a rigid skull protecting against alterations in venous flow, therefore in infants with non-rigid skulls at least a $20 \%$ alteration in cerebral perfusion pressure would be expected. $^{21}$ The fact that a change had definitely occurred was documented by the immediate, presumed passive response in CBFV at the time of the change in posture. In those instances when no immediate change in CBFV was seen, no conclusion on an autoregulatory capacity could be drawn. It is likely that on the occasions when no initial response was seen the posture of the infant was not altered quickly enough. Some of the mattresses had winding mechanisms rather than simple tilting levers and it took longer to alter the infant's posture. It would be incorrect to assume that these infants were autoregulating as no initial stimulus, seen as a change in velocity, has been found to evoke an active autoregulatory response.

The fundamental problem of Doppler ultrasound as a measure of cerebral blood flow is that it is only a measure of CBFV. It is therefore dependent on a constant cross sectional area of the vessel studied to be a true reflection of flow. The cross sectional area of the middle cerebral artery is known to vary in many animal species. ${ }^{28} 303435$ It is theoretically possible that the cross sectional area of the middle cerebral artery could alter such that a 
change in flow occurs with no change in the red cell velocity. This would give a false negative observation, but it is unlikely that the change would be so exact or instantaneous. It is also theoretically possible that a change in velocity may not occur in the same direction as the change in flow if the cross sectional area of the vessel alters. There is evidence from adult studies that changes in velocity recorded from the middle cerebral artery do alter in the same direction as the flow, ${ }^{36}$ but this has not been conclusively shown in neonates. In this study no assumptions are made about the relation of velocity change to flow in the middle cerebral artery. If there is a change in the Doppler velocity, and the probe is fixed at a constant angle of insonation, then some change in the haemodynamics has occurred; the direction of change, an increase or decrease, is not inferred.

We hypothesise that if on altering the infant's posture a change in velocity is recorded, some change in the infant's cerebral haemodynamics has occurred. If no further change in velocity results, then no other change in the circulation has been initiated, but if a second change in velocity occurs this implies a further alteration in the haemodynamics and occurs within the expected time scale of autoregulatory changes.

Systolic velocity readings were used in this study as an indicator of relative changes in cerebral haemodynamics. Diastolic velocities, especially in preterm infants, are often low and difficult to record because of wall thump filters. Loss of the diastolic component alters the mean velocity recorded and therefore the easily obtainable systolic values were used.

This study showed that uniphasic and biphasic responses could be elicited from the same infant, implying that the mechanism for mounting a biphasic response is not an 'all or none' phenomenon. It showed that the reliability of being able to make the biphasic response increased with increasing gestational age. No other physiological or clinical variable was identified which influenced this reliability. The observations made on the infants were made over a limited period of time in proportion to their postnatal ages and it is therefore not surprising that the development of cerebral lesions was not related to the percentage biphasic responses elicited, although it is known that infants of lower gestational ages are more prone to cerebral lesions and it is these infants who less reliably mounted an 'active' response. The $\mathrm{PaCO}_{2}$ and mean arterial pressure did not influence the ability to mount the biphasic response, but the number of infants with high $\mathrm{PaCO}_{2}$ values and low mean arterial pressure was small and no interventions were made to alter these parameters to study their effects directly.

We believe that this study is analysing an autoregulatory response within the strict definition of the term autoregulation. We believe that the biphasic responses witnessed in this study are an indication of an autoregulatory mechanism for the cerebral circulation in neonates. The characteristic of becoming more reliable with increasing gestational age is in keeping with a maturing development.

MYA was supported by the Spastics Society.

1 Volpe JJ. Intraventricular hemorrhage in the preterm infant - current concepts. Part 1. Ann Neurol 1989; 25: 3-11.

2 Pape KE, Wigglesworth JS. Haemorrhage, ischaemia and the prenatal brain. London: Spastics International Medical prenatal brain. London: Spas

3 Lou HC, Lassen NA, Friis-Hansen B. Impaired autoregulation of cerebral blood flow in the distressed newborn infant. F Pediatr 1979; 94: 118-21.

4 Milligan DWA. Failure of autoregulation and intraventricular haemorhage in preterm infants. Lancet 1980; i: 896-8.

5 Ahmann PA, Dykes FD, Lazzara A, Holt PJ, Giddens DP, Carrigan TA. Relationship between pressure passivity and subependymal, intraventricular haemorrhage as 72: 665-9.

6 Jorch G, Jorch N. Failure of autoregulation of cerebral blood flow in neonates studied by pulsed Doppler ultrasound of the internal carotid artery. $\mathcal{F}$ Pediatr 1986; 146: sound of

7 Rosenkrantz TS, Diana D, Munson J. Regulation of cerebral blood flow velocity in non-asphyxiated, very low birth weight infants with hyaline membrane disease. f Perinatol 1988; 8: 303-8.

8 Pryds O, Anderson GE, Friis-Hansen B. Cerebral blood flow reactivity in spontaneously breathing preterm infants shortly after birth. Acta Paediatr Scand 1979; 68: 391-6.

9 Pryds O, Greisen G, Lou H, Friis-Hansen B. Heterogeneity of cerebral vasoreactivity in preterm infants supported by mechanical ventilation. $\mathcal{F}$ Pediatr $1989 ; 115: 638-45$.

10 Heistad DD, Kontos HA. Cerebral circulation. Handbook of physiology - the cardiovascular system. Section 2, Vol 3. physiology - the cardiovascular system. Section 2, Vol 3.

11 Nornes H, Knutgen HB, Wikeby P. Cerebral arterial blood flow and aneurysm surgery. $f$ Neurosurg 1977; 47: 819-27.

12 Busija DW, Heistad DD, Marcus ML. Effects of sympathetic nerves on cerebral vessels during acute, moderate increases in arterial pressure in dogs and cats. Circ Res 1980; 46: 696-702.

13 Strandgaard S, Paulson OB. Progress review - cerebral autoregulation. Stroke 1984; 15: 413-6.

14 Busija DW, Heistad DD. Factors involved in the physiological regulation of the cerebral circulation. Rev Physiol Biochem Pharmacol 1984; 101: 162-211.

15 Fenton A, Evans DH, Levene MI. On-line cerebral blood flow velocity and blood pressure measurement in neonates: a new method. Arch Dis Child 1990; 65: 11-4.

16 Anthony MY, Evans DH, Levene MI. Cyclical variations in cerebral blood flow velocity. Arch Dis Child 1991; 66: $12-6$.

17 Schlindwein FS, Smith MJ, Evans DH. Spectral analysis of Doppler signals and computation of the normalised first movement in real-time using a digital signal processor. Med Biol Eng Comput 1988; 26: 228-32.

18 Fog M. Cerebral circulation. The reaction of the pial arteries to a fall in blood pressure. Arch Neurol Psychol 1937; 37: 353-64.

19 Fog $M$. The relationship between the blood pressure and the tonic regulation of the pial arteries. $\mathcal{F}$ Neurol Psychiatry 1938; 1 : 187-97.

20 Lassen NA. Cerebral blood flow and oxygen consumption in man. Physiological Reviews 1959; 39: 183-238.

21 Smith J, Kampine JP. Cerebral circulation. Circulatory physiology - the essentials. Baltimore: Williams and Wilkins, 1984: 177-216.

22 Gotch F, Ebihara S-I, Toyoda M, Shinohara Y. Role of autonomic nervous system in autoregulation of human cerebral circulation. Eur Neurol 1971-2; 6: 203-7.

23 Paulson OB, Waldemar G. Role of the local reninangiotensin system in the autoregulation of the cerebral angiotensin system in the autoregulation
circulation. Blood Vessels 1991; 28: 231-5.

24 Faraci FM, Heistad DD. Regulation of large cerebral arteries and cerebral microvascular pressure. Circ Res 1990; 66: 8-17.

25 Stromberg DD, Fox JR. Pressure in the pial arterial microcirculation of the cat during changes in systemic arterial blood pressure. Circ Res 1972; XXXI: 229-39.

26 Purves MJ. Control of cerebral blood vessels: present state of the art. Ann Neurol 1978; 3: 377-83.

27 McHenry L, West JW, Cooper ES, Goldberg HI, Jaffe ME. Cerebral autoregulation in man. Stroke 1974; 5: 695-707.

28 Baumbach GL, Heistad DD. Effects of sympathetic stimulation and changes in arterial pressure on segmental resistance of cerebral vessels in rabbits and cats. Circ Res 1983; 52: 527-33.

29 James IM, Millar RA, Purves MJ. Observations on the extrinsic neural control of cerebral blood flow in the baboon. Circ Res 1969; XXV: 77-93.

30 Heistad DD, Marcus ML, Abboud FM. Role of large arteries in regulation of cerebral blood flow in dogs. $\mathcal{f C l i n}$ Invest 1978; 62: 761-8.

31 Busija DW, Heistad DD, Marcus ML. Effects of sympathetic nerves on cerebral vessels during acute, moderate increase in arterial pressure in dogs and cats. Circ Res 1980; 46: 696-702. 
32 Nornes H, Wikeby P. Cerebral arterial blood flow and aneurysm surgery. $\mathcal{F}$ Neurosurg 1977; 47: 810-18.

33 Symen autoregulation in the cerebral circulation to increased autoregulation in the cerebral circulation to increased perfusion pressure in

34 Busija DW, Heistad DD. Effects of cholinergic cerebral blood flow in cats. Circ Res 1981; 48: 62-9.
35 Mchedlishvili G. Principles of cerebral blood flow control a deductive approach. In: Bevan JA, ed. Arterial behavior and blood circulation in the brain. New York: Consultants Bureau, 1985: 17-41

36 Aaslid R, Newell DW, Stooss R, Sorteberg W, Lindegaard K-F. Assessment of cerebral autoregulation dynamics from simultaneous arterial and venous transcranial Doppler recordings in humans. Stroke $1991 ; 22$ : 1148-54. 